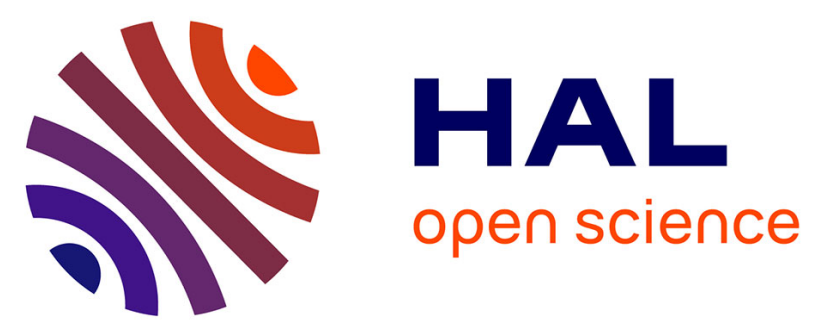

\title{
Regular treadmill exercise restores cardioprotective signaling pathways in obese mice independently from improvement in associated co-morbidities.
}

Sandrine Pons, Valérie Martin, Lolita Portal, Roland Zini, Didier Morin, Alain Berdeaux, Bijan Ghaleh

\section{To cite this version:}

Sandrine Pons, Valérie Martin, Lolita Portal, Roland Zini, Didier Morin, et al.. Regular treadmill exercise restores cardioprotective signaling pathways in obese mice independently from improvement in associated co-morbidities.. Journal of Molecular and Cellular Cardiology, 2013, 54, pp.82-9. 10.1016/j.yjmcc.2012.11.010 . inserm-00796377

\section{HAL Id: inserm-00796377 https://www.hal.inserm.fr/inserm-00796377}

Submitted on 4 Mar 2013

HAL is a multi-disciplinary open access archive for the deposit and dissemination of scientific research documents, whether they are published or not. The documents may come from teaching and research institutions in France or abroad, or from public or private research centers.
L'archive ouverte pluridisciplinaire HAL, est destinée au dépôt et à la diffusion de documents scientifiques de niveau recherche, publiés ou non, émanant des établissements d'enseignement et de recherche français ou étrangers, des laboratoires publics ou privés. 
Regular treadmill exercise restores cardioprotective signalling pathways in obese mice independently from improvement in associated co-morbidities.

Sandrine Pons ${ }^{1,2,4}$; Valérie Martin ${ }^{1,2,4}$; Lolita Portal ${ }^{1,2,4}$; Roland Zini ${ }^{1,2,4}$;

Didier Morin ${ }^{1,2,4}$; Alain Berdeaux ${ }^{1,2,3,4} ;$ Bijan Ghaleh $^{1,2,4}$.

1- Inserm, U955, Equipe 3, Créteil, 94000, France;

2- $\quad$ Université Paris Est, Faculté de Médecine, Créteil, 94000, France;

3- $\quad$ AP-HP, Hôpital H. Mondor - A. Chenevier, Fédération de Cardiologie, Créteil, 94000,

France;

4- Université Paris Est, Ecole Nationale Vétérinaire d'Alfort, Maisons Alfort, 94700,

France.

Corresponding author:

Pr Bijan Ghaleh

INSERM U 955 Equipe 03

Faculté de Médecine

8 rue du Général Sarrail

94000 Créteil

Tel : 33-1-49.81.35.93

Fax : 33-1-48.98.17.77

e-mail: bijan.ghaleh@inserm.fr 


\begin{abstract}
Obesity is a major health issue that impedes the ability of preconditioning and postconditioning to protect the myocardium against infarction secondary to dysregulation of kinase signalling pathways. Moreover, exercise decreases cardiovascular mortality in obese patients but the mechanism remains to be established. Wild-type (WT) and obese (ob/ob) mice were assigned to sedentary conditions or regular treadmill exercise (1h/day, 5 days/7, 4 weeks, $4^{\circ}$ slope, $10-30 \mathrm{~cm} / \mathrm{s}$ ) and underwent $30 \mathrm{~min}$ of coronary artery occlusion followed by $24 \mathrm{~h}$ of reperfusion for infarct size measurement. In WT, exercise reduced infarct size by $60 \%$ and increased phosphorylation of kinases such as Akt, ERK 1/2, p70S6K, AMPK and GSK3ß. Importantly, the level of corresponding phosphatases PTEN, MKP-3 and PP2C was decreased. Calcium concentration inducing opening of mitochondrial permeability transition pore (mPTP) was increased by exercise. In ob/ob, regular exercise induced a robust cardioprotection by reducing infarct size (-67\%), increasing kinase phosphorylation, decreasing phosphatase levels and improving the resistance to mPTP opening. However exercise did not modify hyperglycemia, hypercholesterolemia, hyperinsulinemia, fat mass and body weight in obese mice. In conclusion, regular exercise induces cardioprotection against myocardial infarction despite obesity and restores pro-survival signalling pathways with simultaneous increase in kinase phosphorylations, decreased levels of phosphatases and increased resistance of mPTP opening, independently from improvement in associated comorbidities.
\end{abstract}

Key Words: myocardial infarction, exercise, obesity, kinases, protein phosphatases. 


\section{ABBREVIATIONS}

ACC: acetyl-Co A Carboxylase; Akt: protein kinase B, ERK: extra-cellular signal regulated

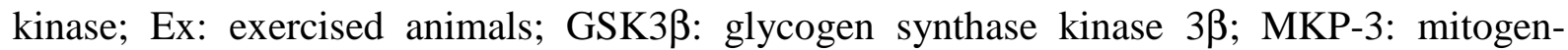
activated protein kinase phosphatase-3, mPTP: mitochondrial permeability transition pore; ob/ob: leptin deficient mice; P-: phosphorylated form; PI3K: phosphatidylinositol-4,5biphosphate 3-kinase; PTEN: Phosphatase and tensin homologue deleted on chromosome 10; PP2C: protein phosphatase 2C, p70S6K : p70S6 kinase; Sed: sedentary. 


\section{INTRODUCTION}

Cardiovascular diseases are a major cause of death in industrialized countries with coronary artery disease being the most prevalent manifestation. Despite reperfusion, coronary artery disease involving myocardial ischemia can result in myocardial infarction associated with elevated morbi-mortality. Consequently, several strategies, pharmacological or not, have been proposed to induce cardioprotection. Among them, regular exercise has been shown to reduce cardiovascular morbi-mortality $[1,2]$ and is considered as the only practical and sustainable countermeasure capable of providing cardioprotection against myocardial infarction. Interestingly, clinical investigations have demonstrated that exercise is a cardioprotective factor, independent of the presence of other risk factors [3-5].

Obesity is also a major health issue in western countries and its importance is constantly growing. According to the World Health Organization, this disease has reached epidemic proportions with more than 1 billion overweight adults and at least 300 million of them are clinically obese. Obesity is a major and independent risk factor for coronary heart disease and increased waist-to-hip ratio is associated with greater occurrence of myocardial infarction [6]. Most animal studies investigating cardioprotective strategies have been performed in young healthy animals but it is well known that co-morbidities such as obesity impede the ability to protect the myocardium against infarction. Indeed it has been previously reported that both pre- and postconditioning failed to reduce infarct size in obese animals probably because of their inability to activate the cardioprotective signalling pathways through kinase phosphorylations. [7-9]. Nevertheless, it is known that exercise is able to decrease cardiac events and mortality in obese or overweight patients [10-12].

In lean conditions, Golbidi et al. [13] have proposed to divide the potential beneficial mechanisms of exercise into two large categories: mechanisms that decrease reactive oxygen 
species production and others that repair cellular damages. Among them, mitochondrial adaptations seem to be crucial in exercise-induced cardioprotection $[14,15]$. Indeed, these organelles, in particular their mitochondrial permeability transition pore (mPTP), represent a major target for protecting the myocardium against infarction [16]. The signalling pathway including PI3K-Akt, ERK 1/2 or GSK3 $\beta$, the so-called Reperfusion Ischemia Salvage Kinases (RISK) pathway has been identified to converge toward the mitochondria and to induce cardioprotection during both pre- and postconditioning [17, 18]. However, this signalling pathway has not been extensively studied for understanding exercise inducedcardioprotection, in particular in obese subjects.

Accordingly, our aim was to investigate whether regular treadmill exercise was able to condition the heart against myocardial infarction through pro-survival signalling pathways during obesity. For this purpose, we investigated in a mouse model of obesity 1) the effects of regular exercise against myocardial infarction and 2) the subsequent activation of the RISK and AMPK pathways as well as mitochondrial adaptations. 


\section{METHODS}

\subsection{Animals}

Male 5 to 10 week-old wild-type C57BL/6J (WT) and obese (ob/ob) mice were used (R. Janvier, Le Genest St Isle, France). Mice were housed in an air-conditioned room with a 12 h light-dark cycle and received standard rodent chow and drinking water ad libitum. The authors have been granted a license from the institutional office "Préfecture du Val de Marne" (France) to conduct animal research (agreement A94-028-28). The investigation conformed to the Guide for the Care and Use of Laboratory Animals published by the US National Institute of Health.

\subsection{Experimental protocol}

Both WT and ob/ob mice were randomly subjected to regular treadmill exercise (Ex-WT and Ex-ob/ob) or to sedentary conditions (Sed-WT and Sed-ob/ob). Exercised animals have run 5/7 days during 4 weeks. The first week was an adaptation period with gradual speeds during $30 \mathrm{~min}\left(10,14,18,22,26\right.$, and $30 \mathrm{~cm} / \mathrm{s}, 4^{\circ}$ slope, 5 min each step for WT and 8, 8, 10, 14,18 , and $20 \mathrm{~cm} / \mathrm{s}, 4^{\circ}$ slope, $5 \mathrm{~min}$ each step for ob/ob). For the three other weeks, mice ran during one hour per day (30 and $20 \mathrm{~cm} / \mathrm{s}$ for WT and ob/ob, respectively, $4^{\circ}$ slope) without any exhaustion. To take into account the differences in body weight, the speed was different between the two strains in order to obtain similar vertical work [19]. After these 4 weeks of exercise or sedentary conditions, mice were subjected to $30 \mathrm{~min}$ of ischemia followed by reperfusion. Infarct sizes were measured at $24 \mathrm{~h}$ of reperfusion. Additional mice were used for Western blot experiments and investigating mPTP opening by calcium. In this case, the heart was excised at $10 \mathrm{~min}$ of reperfusion. All these final experiments were performed $24 \mathrm{~h}$ after 
the last bout of exercise. Additional mice were used for biological and morphological analyses.

\subsection{Morphometry}

Mice were anesthetized by intraperitoneal injection of pentobarbital sodium $(60 \mathrm{mg} / \mathrm{kg})$. Fat and lean masses were determined by means of a PIXImus (Lunar Corporation, Madison, WI, USA) using dual-energy x-ray absorptiometry.

\subsection{Blood measurements}

Blood glucose and cholesterol levels were measured using a multiparametric automat (Automat type AU-400, Olympus, Hamburg, Germany). Insulin level was determined using the Rat/Mouse Insulin ELISA kit (Millipore, Billerica, MA, USA). All these parameters were obtained from fed animals.

\subsection{Myocardial infarction}

Mice were anesthetized by intraperitoneal injection of pentobarbital sodium $(80 \mathrm{mg} / \mathrm{kg})$, intubated and ventilated mechanically. The body temperature was maintained at $37^{\circ} \mathrm{C}$. A left thoracotomy was performed in order to realize the sequence of coronary artery occlusion followed by reperfusion with an 7-0 Prolene thread placed around the left coronary artery as previously described [20]. Myocardial ischemia was confirmed by the occurrence of regional cyanosis. Reperfusion was confirmed by visualisation of an hyperemic response and the chest was closed in layers.

After $24 \mathrm{~h}$ of reperfusion, mice were reanesthetized. The chest was opened, the coronary artery was re-occluded at the same location than previously performed and Evan's blue solution was injected through the apex to delineate the area at risk. The heart was excised, the 
left ventricle was cut into 6 slices and the infarct area was identified by 2,3,5triphenyltetrazolium chloride (TTC) staining. The area at risk was identified as the non-blue region and expressed as a percentage of the left ventricle weight. The infarcted area was identified as the TTC positive zone and expressed as a percentage of area at risk.

\subsection{Mitochondria}

For measurement of mPTP opening, areas at risk were placed in medium containing 220 mM mannitol, 70 mM sucrose, 10 mM HEPES, 1 mM EGTA (Sigma-Aldrich, St. Louis, MO, USA), pH 7.4 at $4{ }^{\circ} \mathrm{C}$. The tissues were minced with scissor and homogenized on ice using a Teflon Potter homogenizer and were centrifuged at $1000 \mathrm{~g}$ for $5 \mathrm{~min}$. Supernatants were centrifuged at $10000 \mathrm{~g}$ for $10 \mathrm{~min}$. The final pellets were resuspended in the homogenization buffer including $0.01 \mathrm{mM}$ EGTA. Protein concentration was determined by the method of Lowry et al. [21].

Mitochondrial $\mathrm{Ca}^{2+}$ retention was monitored as an index of mPTP opening. Mitochondria were loaded with increasing concentrations of $\mathrm{Ca}^{2+}$ until the load reached a threshold where mitochondria underwent a fast process of $\mathrm{Ca}^{2+}$ release due to $\mathrm{mPTP}$ opening. Cardiac mitochondria $(0.8 \mathrm{mg} / \mathrm{ml})$, energized with $3.3 \mathrm{mM}$ pyruvate/malate, were incubated in a buffer $\left(50 \mathrm{mM}\right.$ sucrose, $100 \mathrm{mM} \mathrm{KCl}, 10 \mathrm{mM}$ HEPES and $\left.5 \mathrm{mM} \mathrm{KH}_{2} \mathrm{PO}_{4}\right)$ containing 3.3 $\mu \mathrm{M} \mathrm{Ca}^{2+}$ Green-5N fluorescent probe (Interchim, Montluçon, France). The reaction was started by the addition of successive $2.5 \mu \mathrm{M} \mathrm{Ca}^{2+}$ pulses. After each addition, a rapid uptake was observed and was followed by a dynamic steady state corresponding to the equilibrium between the influx and the efflux of $\mathrm{Ca}^{2+}$. When maximal $\mathrm{Ca}^{2+}$-loading threshold was reached, this equilibrium was disrupted and $\mathrm{Ca}^{2+}$ was released. The concentration of $\mathrm{Ca}^{2+}$ in the extra-mitochondrial medium was monitored by means of a Perkin-Elmer LS 50B 
fluorescence spectrometer (Perkin-Elmer, Waltham, MA, USA) at excitation and emission wavelengths of 506 and $532 \mathrm{~nm}$, respectively.

\subsection{Western Blot analysis}

Areas at risk were placed in medium containing $220 \mathrm{mM}$ mannitol, $70 \mathrm{mM}$ sucrose, 10 $\mathrm{mM}$ HEPES, $1 \mathrm{mM}$ EGTA, $5 \mu \mathrm{l} / \mathrm{ml}$ of protease inhibitor cocktail, $1 \mathrm{mM}$ sodium orthovanadate, $5 \mathrm{mM}$ sodium fluoride, $1 \mathrm{mM}$ sodium $\mathrm{Na}_{2} \beta$-glycerol phosphate (SigmaAldrich, St. Louis, MO, USA), pH 7.4 at $4{ }^{\circ} \mathrm{C}$. The tissues were scissor minced and homogenized on ice using a Teflon Potter homogenizer. The homogenates were centrifuged at $17600 \mathrm{~g}$ for $30 \mathrm{~min}$ at $4^{\circ} \mathrm{C}$ to collect the cytosol. The protein concentrations were determined by the BCA Protein Assay kit (Pierce, Rockford, IL, USA).

Extracted protein samples were denatured at $90^{\circ} \mathrm{C}$ for $5 \mathrm{~min}$. Proteins $(25 \mu \mathrm{g} / \mathrm{lane})$ were separated by SDS-polyacrylamide gel electrophoresis, transferred to PVDF membranes and probed with primary antibodies for phospho-Akt (Serine 473), phospho-ERK 1/2 (Threonine 202/Tyrosine 204), phospho-p70S6K (Threonine 389), phospho-p70S6K (Threonine 421/Serine 424), phospho-AMPK (Threonine 172), phospho-GSK3 $\beta$ (Serine 9), phosphoACC (Serine 79), total Akt, total ERK1/2, total p70S6K, total AMPK, total GSK3ß, total ACC, PTEN (all from Cell Signaling Technology, Danvers, MA, USA), MKP-3 (Epitomics, Burlingame, CA, USA), PP2C (Abcam, Cambridge, UK). Blots were washed and then incubated with goat anti-rabbit IgG secondary antibody conjugated to horseradish peroxidase (Santa Cruz Biotechnology, Santa Cruz, CA, USA). Blots were finally incubated using the ECL detection system (Pierce, Rockford, IL, USA) and exposed to ECL chemiluminescence film (Hyperfilm ECL, Amersham, Buckinghamshire, UK). Bands of interest were scanned and quantified in a blinded manner using gel analysis software Image J-1.37 (National Institute of Health, Bethesda, MD, USA). 
2.8. Statistical analysis

All values were expressed as mean \pm SEM. Comparisons were performed using a Kruskal-Wallis analysis followed by Mann-Whitney tests. Statistical significance was defined as a value of $p<0.05$. 


\section{RESULTS}

\subsection{Morphological and biological parameters}

As shown in Table 1, body weights and fat masses were increased by 1.7-fold and 4.3fold respectively in ob/ob as compared to WT sedentary mice $(\mathrm{p}<0.05)$. Blood glucose, cholesterol and insulin levels were significantly higher in ob/ob as compared to WT sedentary mice. Regular exercise had no effect on these three biological parameters either in ob/ob or in WT animals. In addition, body weights and fat masses were not affected by regular exercise.

\subsection{Myocardial infarct sizes}

The areas at risk were similar among all groups $(41 \pm 1 \%, 41 \pm 3 \%, 38 \pm 1 \%$ and $38 \pm 3 \%$ for Sed-WT, Ex-WT, Sed-ob/ob and Ex-ob/ob, respectively). Infarct size was significantly increased in ob/ob vs WT sedentary mice (Figure 1). In exercised WT mice, infarct size was reduced by $60 \%(43 \pm 3 \%$ vs $17 \pm 2 \%$ for Sed-WT and Ex-WT, respectively, $\mathrm{p}<0.05)$. In ob/ob mice, regular exercise elicited a major cardioprotective effect as infarct size was significantly reduced by $67 \%$ (58 $\pm 3 \%$ vs $19 \pm 1 \%$ for Sed-ob/ob and Ex-ob/ob, respectively).

\subsection{Signalling pathways}

In exercised WT mice (Figure 2), the phospho-Akt/Akt ratio was significantly increased after ischemia-reperfusion. As p70S6K is known to be phosphorylated at Threonine 389 through the Akt pathway, we examined its phosphorylation. Western blot revealed a raise in phosphorylated $\mathrm{p} 70 \mathrm{~S} 6 \mathrm{~K} / \mathrm{p} 70 \mathrm{~S} 6 \mathrm{~K}$ ratio by exercise $(+65 \%, \mathrm{p}<0.05)$. Concomitantly, PTEN which participates to Akt inactivation was significantly decreased in exercised as compared to sedentary animals. Interestingly, all these effects were also observed in ob/ob mice. 
As illustrated in Figure 3, exercised WT mice elicited major increases in the phosphorylated form of ERK $1 / 2$ and of one of its downstream kinase, i.e., p70S6K (Thr 421/Ser 424) while the ERK $1 / 2$ corresponding phosphatase MKP3 levels were simultaneously reduced as compared to sedentary WT mice. In ob/ob animals, exercise allowed once again to induce major significant increases in the level of these phosphorylated kinases and decrease in phosphatase levels.

Then we examined AMPK that plays a critical role in maintaining energy homeostasis and cardiac function during stress situations such as ischemia (Figure 4). We found that phosphorylation of AMPK and one of its characterized targets ACC, were significantly increased after ischemia-reperfusion in exercised as compared to sedentary WT mice. Concomitantly, levels of PP2C which inactivates AMPK, were significantly reduced. Similar pattern of phosphorylation and phosphatase variation was observed in exercised $v s$ sedentary ob/ob animals.

We also investigated GSK3 $\beta$, one of the downstream target of ERK 1/2 and Akt signalling pathways that is known to play a role in mPTP opening (Figure 5A). We found that phospho-GSK3 $3 /$ GSK3 $\beta$ ratio was significantly increased by exercise. In ob/ob animals, regular exercise also induced significant and similar increase in phosphorylation of GSK3 $\beta$. Finally, we evaluated the adaptation in mPTP opening by assessing its susceptibility to calcium overload. The $\mathrm{Ca}^{2+}$ concentration required to open mPTP was significantly improved by $95 \%$ in exercised as compared to sedentary WT mice (Figure 5B). Improved resistance to mPTP opening was also observed in exercised ob/ob animals. 


\section{DISCUSSION}

The present study demonstrates that regular treadmill exercise induces protection against myocardial infarction despite obesity and activates cellular cardioprotective kinases, independently from improvement in associated co-morbidities. Therefore, regular exercise is a powerful cardioprotective strategy that restores the activation of pro-survival signalling pathways and protects cardiac mitochondria. This is of major importance as both pre- and postconditioning failed to reduce myocardial infarct size in obese animals because of their inability to activate these cardioprotective pathways in obese conditions (for review, [8]). Importantly, the variations in phosphatase levels mirrored those of kinase phosphorylations as the increased amount of phosphorylated forms of survival kinases was accompanied by a reduction in phosphatases expression, i.e., PTEN, MKP-3 and PP2C. The mechanisms of this protection also involved major cardiac mitochondrial adaptations illustrated by an increased capacity of mitochondria to retain calcium showing a greater resistance to $\mathrm{mPTP}$ opening.

In the present study, we used the ob/ob mice model of obesity characterized by hyperphagia with increased body weight and metabolic abnormalities. With regular exercise, we did not however observe any significant changes in body weight, fat mass, glycemia, cholesterolemia and insulinemia, probably related to maintained hyperphagia although individual food intake was not measured in the present study. This is an important feature of our experimental setting that allowed to avoid any confounding effect induced by changes in these biological parameters. This latter point is in agreement with previous studies. MoienAfshari et al. [22] have shown that exercise restores coronary vascular function independently of changes in hyperglycaemic and hyperinsulinemic status in $\mathrm{db} / \mathrm{db}$ mice. In another study, treadmill exercise failed to restore body weight in the $\mathrm{db} / \mathrm{db}$ mouse model [23]. Therefore, 
exercise induced-cardioprotection in these obese animals was independent from any improvement in associated co-morbidities.

Numerous experimental studies have reported cardioprotection with exercise and it has been proposed that it could improve myocardial antioxidant capacity, increase cardiac ATPsensitive potassium channel expression, prevent calpaïn activation and decrease calcium overload [24-27]. To our knowledge, although several pro-survival kinases and mitochondria are described as major actors of cardioprotection [17, 18], no study has investigated their role in exercise-trained animals, either healthy or obese, during myocardial ischemia-reperfusion. Only Zhang et al [28] have described that long term aerobic exercise alleviated cardiac dysfunction following myocardial infarction through a PI3K-Akt and GSK3 $\beta$ dependent mechanism in rats. One major insight of the present study is the concomitant investigation of the RISK pathway as well as its downstream target, the mitochondria but also AMPK and several phosphatases. Indeed following ischemia-reperfusion in WT and ob/ob mice, we observed that regular exercise induced activation of the RISK pathway through phosphorylation of its components, i.e., Akt, ERK 1/2 and their common downstreams, p70S6K and GSK 3 $\beta$. In parallel, the variations of phosphatase levels mirrored those of corresponding kinase phosphorylations as PTEN and MKP-3 were concomitantly decreased by regular exercise. This point is of major importance as it is known that phosphatases limit the efficacy of some cardioprotective strategies such as pre- or postconditioning during aging or obesity $[9,29,30]$. One could suggest that reduced phosphatases expression would be responsible for the activation of survival kinases which is known to be a crucial event for protecting the myocardium against myocardial infarction. Indeed, it has been clearly demonstrated for other cardioprotective strategy that phosphorylation of kinases at reperfusion is essential for inducing cardioprotection independently from cellular events that could occur prior to reperfusion [31]. Blockade experiments were not performed and will 
require other studies. Moreover, the use of inhibitors of ERK and Akt pathways might be complicated as compensatory mechanisms and crosstalks between signalling pathways could exist.

Activation of these signalling pathways is well known to target mitochondria by protecting against mPTP opening and ultimately from cell death. Indeed, the critical role of mPTP has been demonstrated during cardioprotection elicited by ischemic and pharmacological pre- and post-conditioning $[16,18]$. Accordingly, we evidenced that regular exercise increased the capacity of mitochondria to retain calcium in WT but also similarly in obese animals during ischemia-reperfusion. These findings are in accordance with the prevention of the release of proapoptotic proteins from mitochondria by treadmill exercise [32]. Another point deserves to be discussed. In sedentary animals, myocardial infarct sizes were greater in ob/ob vs WT mice while the capacity of mitochondria to retain calcium was similar. This apparent discrepancy might be related to the difference in calcium handling between WT and ob/ob mice and therefore to a greater level of mPTP opening stimulation by calcium in the cardiomyocyte. Indeed, calcium leak from sarcoplasmic reticulum through the ryanodine channel and altered calcium reuptake could occur in ob/ob as previously reported in $\mathrm{db} / \mathrm{db}$ mice, these alterations being normalized by exercise [33].

We also investigated AMPK that plays a critical role in maintaining energy homeostasis and cardiac function during stress situations such as ischemia. Indeed AMPK plays an important protective role in limiting damage associated with ischemia-reperfusion in the heart [34]. Exercised mice exhibited increased phosphorylations at Thr172 of AMPK and of one of its characterized targets ACC. AMPK activation may protect the heart through improved preservation of cardiac energetics. Once again, this activation was observed in both strains. These results are in agreement with previous findings demonstrating that improving AMPK activation protects the diabetic heart against ischemia-reperfusion [35]. Similarly to the 
balance between kinases and phosphatases for the RISK pathway, the phosphatase PP2C level, which is known to inactivate AMPK, was reduced while the phosphorylated form of AMPK was increased in exercised mice.

Other aspects deserve to be discussed. We can hypothesize that a small amount of reactive oxygen species produced during each bout of exercise may trigger the cardiac adaptations. Indeed, studies have demonstrated that cardioprotective effects of exercise or tachycardia were abolished when anti-oxydants are given during exercise [36-38]. Furthermore, reactive oxygen species are able to stimulate the PI3K-Akt, ERK1/2 and AMPK pathways [39-41]. This might partly explain potential beneficial adaptations of calcium handling with exercise. Indeed, it has been previously reported that exercise increases ryanodine-S-glutathionylation which inhibits ryanodine-mediated calcium leak [38].

\section{CONCLUSIONS}

Independently from improvement in associated co-morbidities, regular treadmill exercise induces cardioprotection against myocardial infarction in obese mice. Despite obesity, exercise is able to restore similar capacity to lean animal at activating pro-survival signalling pathways, contrasting with other conditioning strategies. This mechanism might participate to the beneficial effects of regular exercise against myocardial events in obese or overweight patients. 


\section{SOURCES OF FUNDING}

This study was supported by a grant from the Fondation de France (2008-002688).

Lolita Portal was a recipient of a grant from the Region Ile de France (CODDIM, 2010).

\section{ACKNOWLEDGEMENTS}

The authors are greatly indebted to Dr M. Longchampt (Institut de Recherche Servier) for dual-energy x-ray absorptiometry measurements and to the laboratoire de biochimie (ICBIFR2, Université Paris Diderot-Paris 7) for blood analyses.

\section{DISCLOSURES}

None declared. 


\section{REFERENCES}

1. Sofi F, Capalbo A, Cesari F, Abbate R, Gensini GF. Physical activity during leisure time and primary prevention of coronary heart disease: an updated meta-analysis of cohort studies. Eur J Cardiovasc Prev Rehabil 2008; 15: 247-57.

2. Thompson PD, Buchner D, Pina IL, Balady GJ, Williams MA, Marcus BH et al. Exercise and physical activity in the prevention and treatment of atherosclerotic cardiovascular disease: a statement from the Council on Clinical Cardiology (Subcommittee on Exercise, Rehabilitation, and Prevention) and the Council on Nutrition, Physical Activity, and Metabolism (Subcommittee on Physical Activity). Circulation 2003; 107: 3109-16.

3. Bijnen FC, Caspersen CJ, Mosterd WL. Physical inactivity as a risk factor for coronary heart disease: a WHO and International Society and Federation of Cardiology position statement. Bull World Health Organ 1994; 72: 1-4.

4. Blair SN, Kampert JB, Kohl HW, 3rd, Barlow CE, Macera CA, Paffenbarger RS, Jr. et al. Influences of cardiorespiratory fitness and other precursors on cardiovascular disease and allcause mortality in men and women. Jama 1996; 276: 205-10.

5. Fletcher GF, Blair SN, Blumenthal J, Caspersen C, Chaitman B, Epstein S et al. Statement on exercise. Benefits and recommendations for physical activity programs for all Americans. A statement for health professionals by the Committee on Exercise and Cardiac Rehabilitation of the Council on Clinical Cardiology, American Heart association. Circulation 1992; 86: $340-4$. 
6. Yusuf S, Hawken S, Ounpuu S, Bautista L, Franzosi MG, Commerford P et al. Obesity and the risk of myocardial infarction in 27,000 participants from 52 countries: a case-control study. Lancet 2005; 366: 1640-9.

7. Ferdinandy $\mathrm{P}$, Schulz R, Baxter GF. Interaction of cardiovascular risk factors with myocardial ischemia/reperfusion injury, preconditioning, and postconditioning. Pharmacol Rev 2007; 59: 418-58.

8. Peart JN, Headrick JP. Clinical cardioprotection and the value of conditioning responses. Am J Physiol Heart Circ Physiol 2009; 296: H1705-20.

9. Bouhidel O, Pons S, Souktani R, Zini R, Berdeaux A, Ghaleh B. Myocardial ischemic postconditioning against ischemia-reperfusion is impaired in ob/ob mice. Am J Physiol Heart Circ Physiol 2008; 295: H1580-6.

10. Blair SN, Brodney S. Effects of physical inactivity and obesity on morbidity and mortality: current evidence and research issues. Med Sci Sports Exerc 1999; 31: S646-62.

11. Savage PD, Brochu M, Poehlman ET, Ades PA. Reduction in obesity and coronary risk factors after high caloric exercise training in overweight coronary patients. Am Heart J 2003; 146: 317-23.

12. Singh RB, Rastogi V, Rastogi SS, Niaz MA, Beegom R. Effect of diet and moderate exercise on central obesity and associated disturbances, myocardial infarction and mortality in patients with and without coronary artery disease. J Am Coll Nutr 1996; 15: 592-601.

13. Golbidi S, Laher I. Molecular mechanisms in exercise-induced cardioprotection. Cardiol Res Pract 2011; 2011: 972807. 
14. Kavazis AN, Alvarez S, Talbert E, Lee Y, Powers SK. Exercise training induces a cardioprotective phenotype and alterations in cardiac subsarcolemmal and intermyofibrillar mitochondrial proteins. Am J Physiol Heart Circ Physiol 2009; 297: H144-52.

15. Kavazis AN, McClung JM, Hood DA, Powers SK. Exercise induces a cardiac mitochondrial phenotype that resists apoptotic stimuli. Am J Physiol Heart Circ Physiol 2008; 294: H928-35.

16. Lim SY, Davidson SM, Hausenloy DJ, Yellon DM. Preconditioning and postconditioning: the essential role of the mitochondrial permeability transition pore. Cardiovasc Res 2007; 75: $530-5$.

17. Hausenloy DJ, Lecour S, Yellon DM. Reperfusion injury salvage kinase and survivor activating factor enhancement prosurvival signaling pathways in ischemic postconditioning: two sides of the same coin. Antioxid Redox Signal 2011; 14: 893-907.

18. Heusch G, Boengler K, Schulz R. Cardioprotection: nitric oxide, protein kinases, and mitochondria. Circulation 2008; 118: 1915-9.

19. Massett MP, Berk BC. Strain-dependent differences in responses to exercise training in inbred and hybrid mice. Am J Physiol Regul Integr Comp Physiol 2005; 288: R1006-13.

20. Michael LH, Ballantyne CM, Zachariah JP, Gould KE, Pocius JS, Taffet GE et al. Myocardial infarction and remodeling in mice: effect of reperfusion. Am J Physiol 1999; 277: H660-8.

21. Lowry OH, Rosebrough NJ, Farr AL, Randall RJ. Protein measurement with the Folin phenol reagent. J Biol Chem 1951; 193: 265-75. 
22. Moien-Afshari F, Ghosh S, Elmi S, Khazaei M, Rahman MM, Sallam N et al. Exercise restores coronary vascular function independent of myogenic tone or hyperglycemic status in db/db mice. Am J Physiol Heart Circ Physiol 2008; 295: H1470-80.

23. Sennott J, Morrissey J, Standley PR, Broderick TL. Treadmill exercise training fails to reverse defects in glucose, insulin and muscle GLUT4 content in the $\mathrm{db} / \mathrm{db}$ mouse model of diabetes. Pathophysiology 2008; 15: 173-9.

24. Brown DA, Lynch JM, Armstrong CJ, Caruso NM, Ehlers LB, Johnson MS et al. Susceptibility of the heart to ischaemia-reperfusion injury and exercise-induced cardioprotection are sex-dependent in the rat. J Physiol 2005; 564: 619-30.

25. Domenech R, Macho P, Schwarze H, Sanchez G. Exercise induces early and late myocardial preconditioning in dogs. Cardiovasc Res 2002; 55: 561-6.

26. French JP, Hamilton KL, Quindry JC, Lee Y, Upchurch PA, Powers SK. Exerciseinduced protection against myocardial apoptosis and necrosis: MnSOD, calcium-handling proteins, and calpain. Faseb J 2008; 22: 2862-71.

27. Soufi FG, Saber MM, Ghiassie R, Alipour M. Role of 12-week resistance training in preserving the heart against ischemia-reperfusion-induced injury. Cardiol J 2011; 18: 140-5.

28. Zhang KR, Liu HT, Zhang HF, Zhang QJ, Li QX, Yu QJ et al. Long-term aerobic exercise protects the heart against ischemia/reperfusion injury via PI3 kinase-dependent and Aktmediated mechanism. Apoptosis 2007; 12: 1579-88.

29. Fenton RA, Dickson EW, Dobson JG, Jr. Inhibition of phosphatase activity enhances preconditioning and limits cell death in the ischemic/reperfused aged rat heart. Life Sci 2005; 77: $3375-88$ 
30. Przyklenk K, Maynard M, Darling CE, Whittaker P. Aging mouse hearts are refractory to infarct size reduction with post-conditioning. J Am Coll Cardiol 2008; 51: 1393-8.

31. Hausenloy DJ, Tsang A, Mocanu MM, Yellon DM. Ischemic preconditioning protects by activating prosurvival kinases at reperfusion. Am J Physiol Heart Circ Physiol 2005; 288: H971-6.

32. Lee Y, Min K, Talbert EE, Kavazis AN, Smuder AJ, Willis WT et al. Exercise protects cardiac mitochondria against ischemia-reperfusion injury. Med Sci Sports Exerc 2012; 44: $397-405$

33. Stolen TO, Hoydal MA, Kemi OJ, Catalucci D, Ceci M, Aasum E et al. Interval training normalizes cardiomyocyte function, diastolic $\mathrm{Ca} 2+$ control, and $\mathrm{SR} \mathrm{Ca} 2+$ release synchronicity in a mouse model of diabetic cardiomyopathy. Circ Res 2009; 105: 527-36.

34. Russell RR, 3rd, Li J, Coven DL, Pypaert M, Zechner C, Palmeri M et al. AMP-activated protein kinase mediates ischemic glucose uptake and prevents postischemic cardiac dysfunction, apoptosis, and injury. J Clin Invest 2004; 114: 495-503.

35. Paiva MA, Rutter-Locher Z, Goncalves LM, Providencia LA, Davidson SM, Yellon DM et al. Enhancing AMPK activation during ischemia protects the diabetic heart against reperfusion injury. Am J Physiol Heart Circ Physiol 2011; 300: H2123-34.

36. Akita Y, Otani H, Matsuhisa S, Kyoi S, Enoki C, Hattori R et al. Exercise-induced activation of cardiac sympathetic nerve triggers cardioprotection via redox-sensitive activation of eNOS and upregulation of iNOS. Am J Physiol Heart Circ Physiol 2007; 292: H2051-9. 
37. Lucats L, Chalvignac V, Bize A, Monnet X, Zini R, Hittinger L et al. Rapid ventricular pacing induces delayed cardioprotection against myocardial stunning. J Mol Cell Cardiol 2005; 39: 849-55.

38. Sanchez G, Escobar M, Pedrozo Z, Macho P, Domenech R, Hartel S et al. Exercise and tachycardia increase NADPH oxidase and ryanodine receptor-2 activity: possible role in cardioprotection. Cardiovasc Res 2008; 77: 380-6.

39. Barbosa VA, Luciano TF, Marques SO, Vitto MF, Souza DR, Silva LA et al. Acute exercise induce endothelial nitric oxide synthase phosphorylation via Akt and AMP-activated protein kinase in aorta of rats: Role of reactive oxygen species. Int J Cardiol 2012. in press.

40. Clerk A, Michael A, Sugden PH. Stimulation of multiple mitogen-activated protein kinase sub-families by oxidative stress and phosphorylation of the small heat shock protein, HSP25/27, in neonatal ventricular myocytes. Biochem J 1998; 333 ( Pt 3): 581-9.

41. Nelson MJ, Harris MB, Boluyt MO, Hwang HS, Starnes JW. Effect of N-2mercaptopropionyl glycine on exercise-induced cardiac adaptations. Am J Physiol Regul Integr Comp Physiol 2011; 300: R993-R1000. 


\section{FIGURE LEGENDS}

\section{Figure 1}

Infarct size (expressed as percentage of the area at risk) measured in wild-type (WT) and $\mathrm{ob} / \mathrm{ob}$ mice subjected to $30 \mathrm{~min}$ of coronary artery occlusion and $24 \mathrm{~h}$ of reperfusion in exercise (Ex) or sedentary (Sed) conditions. Open circles represent individual values and closed circles indicate the average. ${ }^{*} p<0.05 v s$ corresponding sedentary group. $\dagger p<0.05 v s$ corresponding WT group.

\section{Figure 2}

Western blot analysis of Akt and its phosphorylated form (panel A), p70S6K and its phosphorylated form at Thr 389 due to the effect of Akt (panel B) as well as PTEN (panel C) in wild-type (WT) and ob/ob mice in sedentary conditions (Sed) or after regular exercise (Ex). Values are expressed as mean \pm SEM ( $\mathrm{n}=3$ in WT groups and 4 in ob/ob groups). $\quad * p<0.05$ $v s$ corresponding sedentary group. $\dagger p<0.05 v s$ corresponding WT group.

\section{Figure 3}

Western blot analysis of ERK 1/2 and its phosphorylated form (panel A), p70S6K and its phosphorylated form at Thr 421 and Ser 424 due to the effects of ERK1/2 (panel B) as well as MKP-3 (panel C) in wild-type (WT) and ob/ob mice in sedentary conditions (Sed) or after regular exercise (Ex). Values are expressed as mean \pm SEM $(n=3$ in WT groups and 4 in ob/ob groups). ${ }^{*} p<0.05 v s$ corresponding sedentary group. $\dagger p<0.05$ vs corresponding WT group. 


\section{Figure 4}

Western blot analysis of AMPK, ACC and their phosphorylated forms (panels A and B) as well as PP2C (panel C) in wild-type (WT) and ob/ob mice in sedentary conditions (Sed) or after regular exercise (Ex). Values are expressed as mean \pm SEM ( $n=3$ in WT groups and 4 in ob/ob groups). $* p<0.05$ vs corresponding sedentary group. $\dagger p<0.05$ vs corresponding WT group.

\section{Figure 5}

Western blot analysis of GSK3 $\beta$ and its phosphorylated form (panel A, n=3 in WT groups and $4 \mathrm{in} \mathrm{ob/ob}$ groups) as well as mitochondrial $\mathrm{Ca}^{2+}$ retention (index of mPTP opening) (panel $\mathrm{B}$, $\mathrm{n}=6$-10) in wild-type (WT) and ob/ob mice in sedentary conditions (Sed) or after regular exercise (Ex). Representative tracings of calcium-induced mPTP opening in exercised (a) and sedentary (b) ob/ob mice (panel C). Values are expressed as mean \pm SEM. $* p<0.05$ vs corresponding sedentary group. $\dagger p<0.05 v s$ corresponding WT group. 
Table 1. Morphological and biological parameters

\begin{tabular}{lrrrrr}
\hline & \multicolumn{2}{c}{$W T$} & & \multicolumn{2}{c}{ ob/ob } \\
\cline { 2 - 3 } \cline { 5 - 6 } & \multicolumn{1}{c}{ Sed } & Ex & & Sed & Ex \\
\hline Body weight, g & $25 \pm 0.6$ & $24.2 \pm 1.0$ & & $42.8 \pm 0.6 \dagger$ & $44.8 \pm 0.7 \dagger$ \\
Fat mass, \% body weight & $12.5 \pm 1.0$ & $12.6 \pm 0.6$ & & $53.2 \pm 0.5 \dagger$ & $55.6 \pm 0.6 \dagger$ \\
Glycemia (mmol/L) & $10.3 \pm 0.6$ & $11.7 \pm 1.1$ & & $18.8 \pm 2.5 \dagger$ & $17.5 \pm 2.1 \dagger$ \\
Cholesterolemia (mmol/L) & $2.4 \pm 0.1$ & $2.3 \pm 0.1$ & & $5.0 \pm 0.5 \dagger$ & $4.9 \pm 0.6 \dagger$ \\
Insulinemia (ng/mL) & $0.9 \pm 0.2$ & $1.2 \pm 0.4$ & & $12.9 \pm 2.9 \dagger$ & $13.9 \pm 3.5 \dagger$ \\
\hline
\end{tabular}

Values are mean \pm s.e.m. $\mathrm{n}=5$ to 10 .

$\dagger, \mathrm{p}<0.05 v s$ corresponding WT group. 


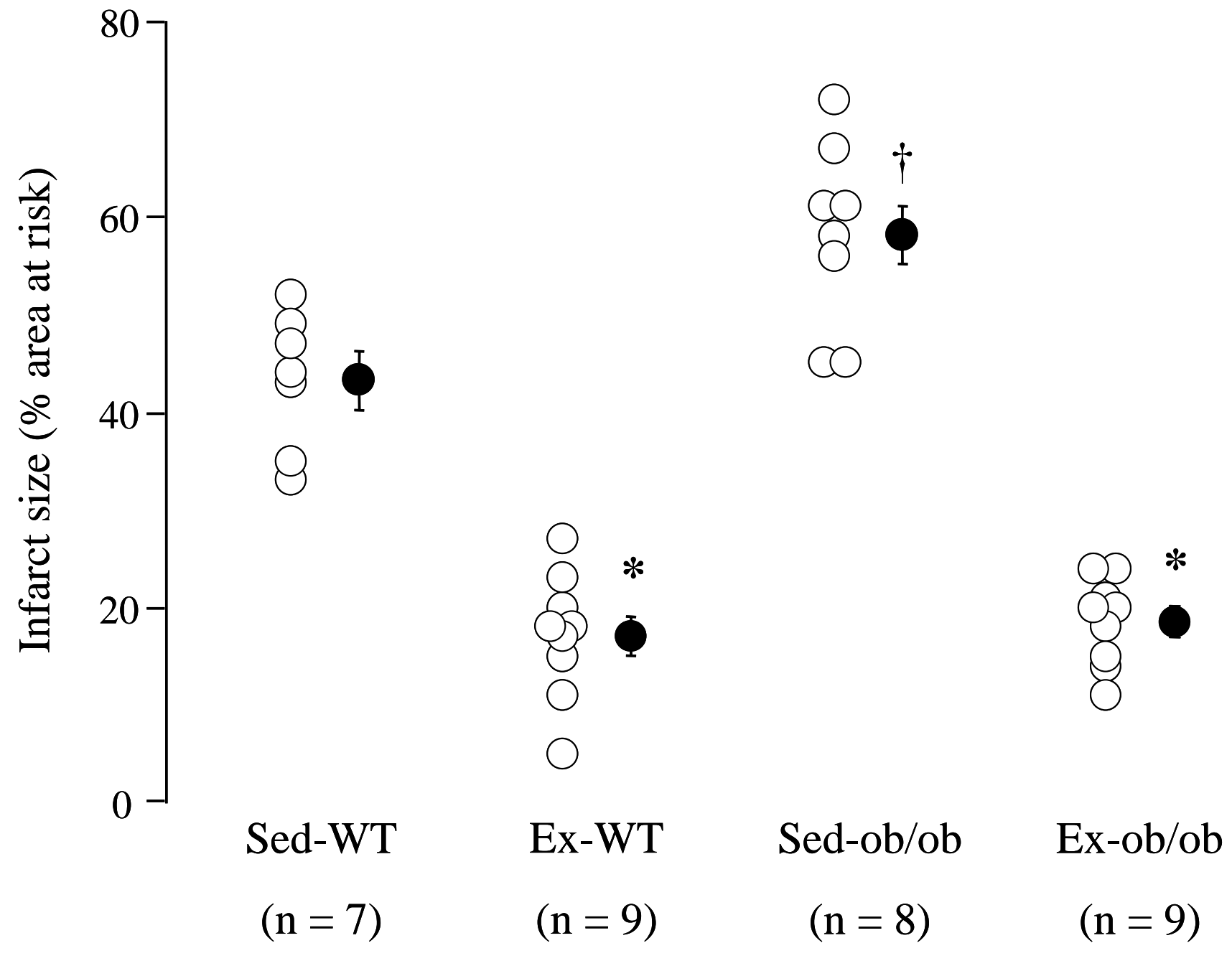




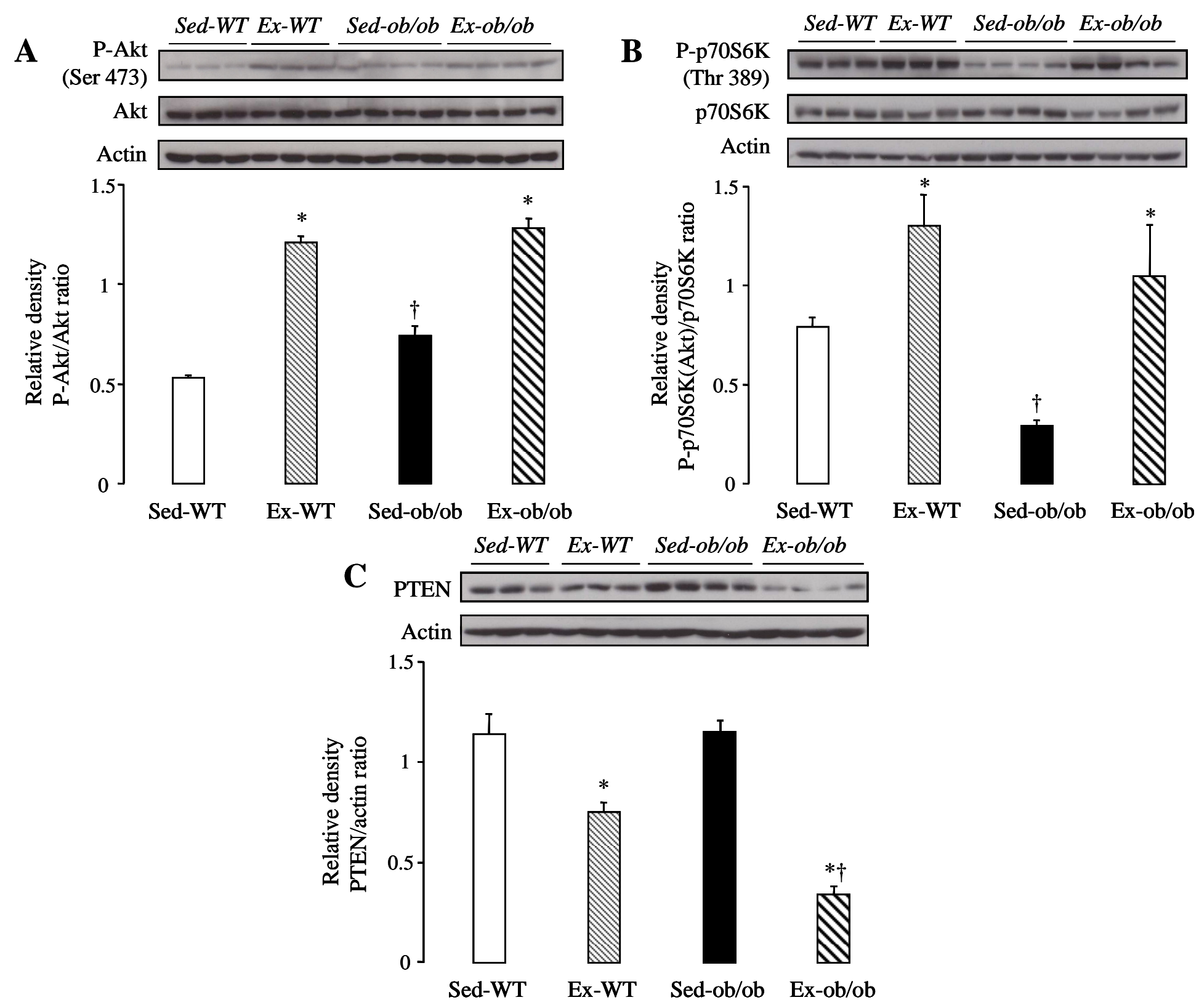




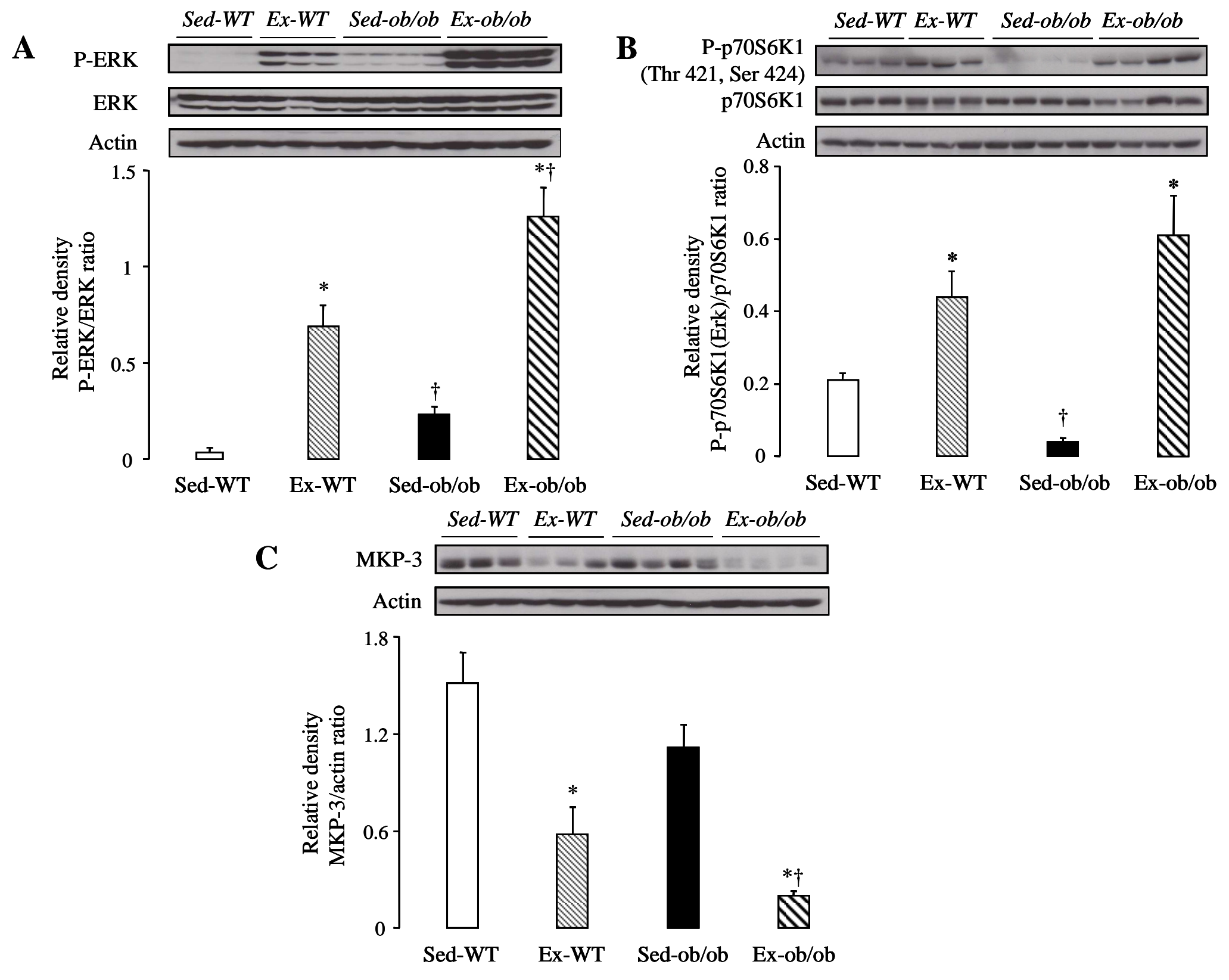




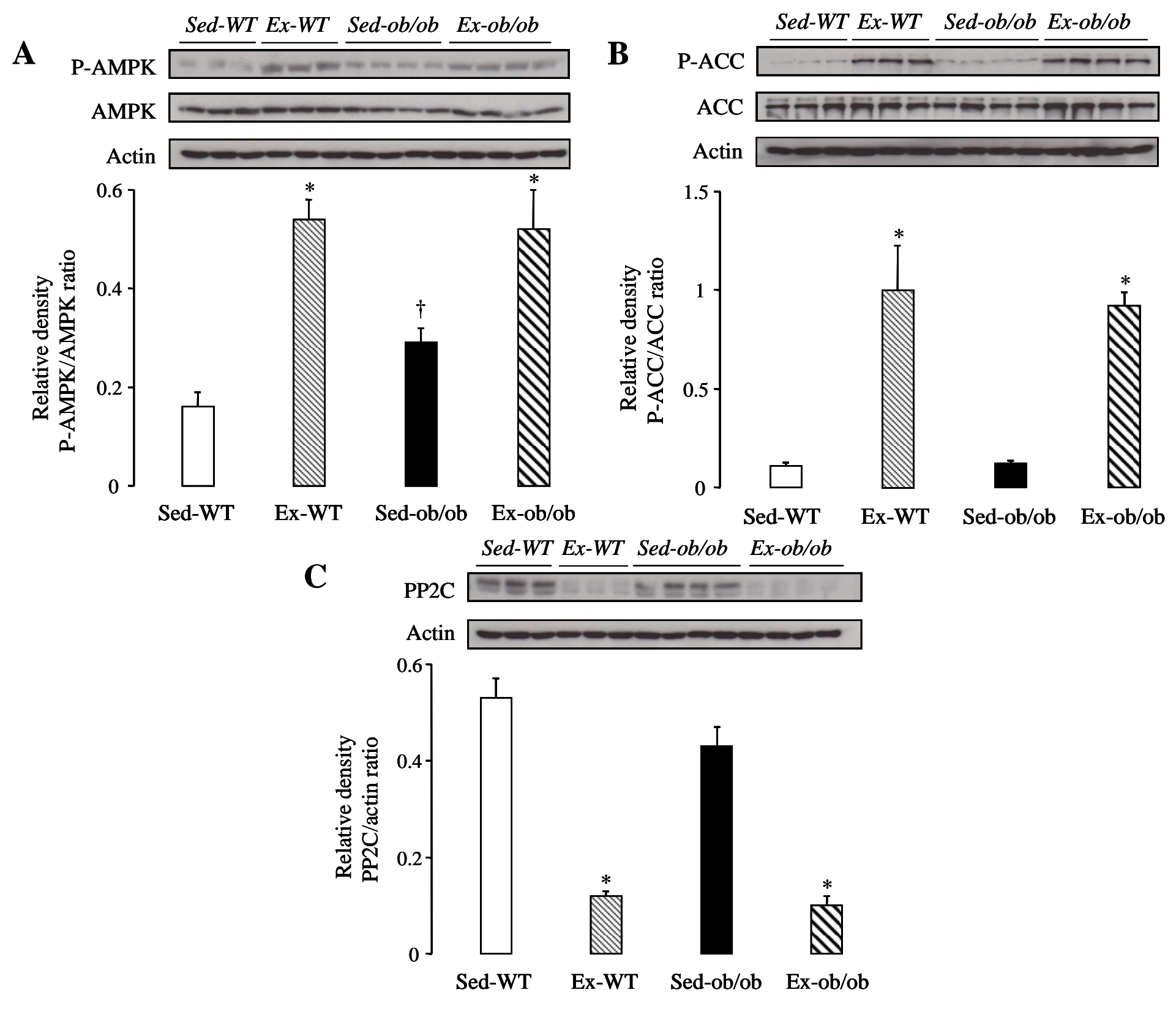



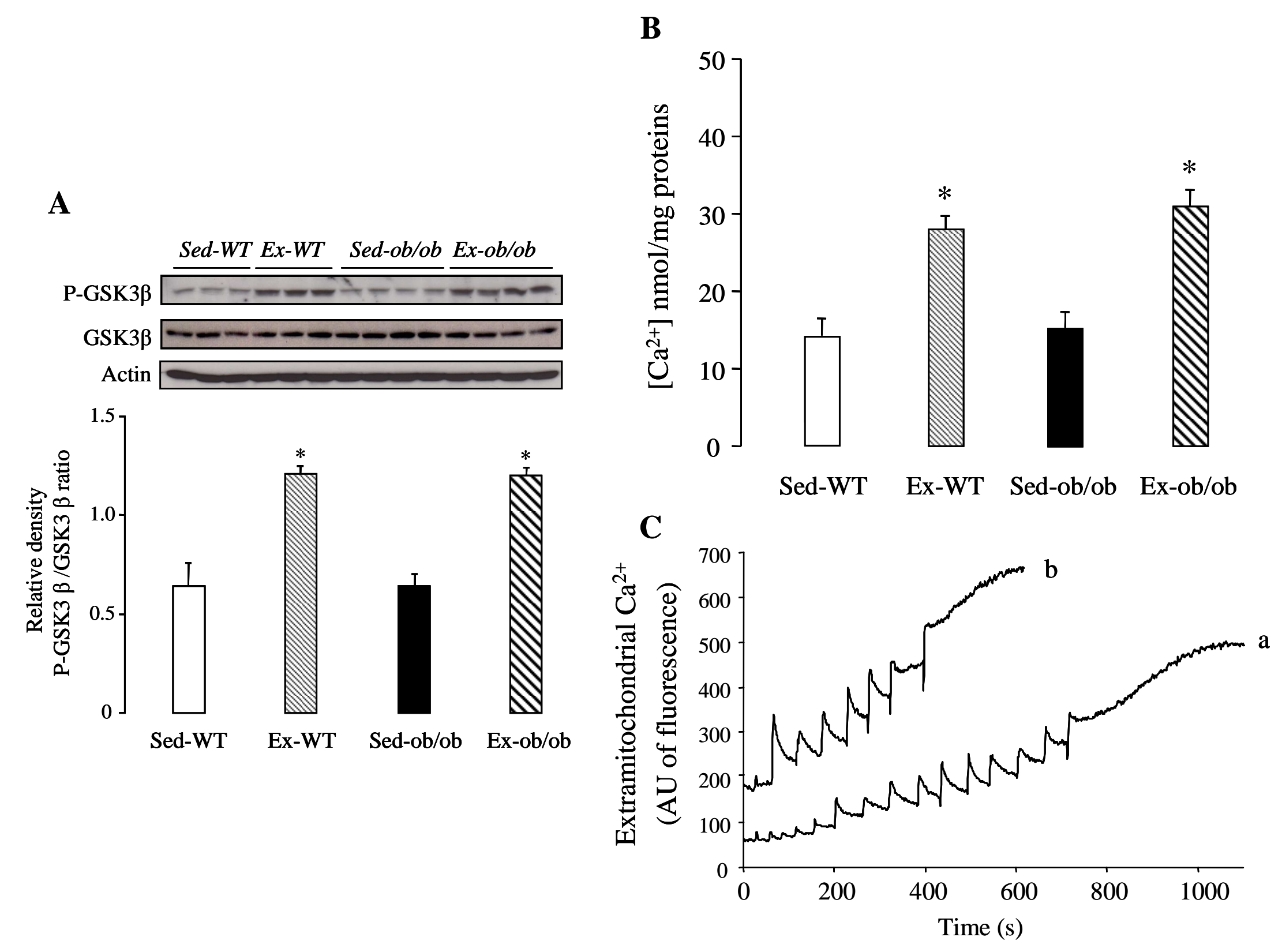